\title{
A Unified Theoretical Model for the Monotonic and Cyclic Response of FRP Strips Glued to Concrete
}

\author{
Enzo Martinelli ${ }^{1, *}$ and Antonio Caggiano ${ }^{2}$
}

1 Department of Civil Engineering, University of Salerno, via Giovanni Paolo II, 84084 Fisciano (SA), Italy

2 CONICET (Argentinean National Council of Scientific and Technical Research) and University of Buenos Aires, Av. Las Heras 2214, C1127AAR CABA, Argentina; E-Mail: acaggiano@fi.uba.ar

* Author to whom correspondence should be addressed; E-Mail: e.martinelli@unisa.it; Tel.: +39-089-96-4098; Fax: +39-089-96-4045.

Received: 30 November 2013; in revised form: 20 January 2014 / Accepted: 27 January 2014 / Published: 3 February 2014

\begin{abstract}
The mechanical behavior of the adhesive interface between the fiber-reinforced polymer (FRP) strip and the concrete substrate often controls the response of FRP-strengthened reinforced concrete (RC) members. Plenty of studies devoted to understanding the mechanical behavior of FRP strips glued to concrete are currently available in the scientific literature. However, they are mainly focused on the response under monotonic actions, which is certainly relevant in a wide class of practical applications. Conversely, few contributions are currently available to better understand the response of FRP-to-concrete interfaces under cyclic actions, such as those deriving from either seismic excitations or traffic loads. This paper presents a unified numerical approach to simulate both monotonic and cyclic behavior of FRP plates glued on quasi-brittle substrates like those made of concrete. Particularly, a damage-based approach is proposed to simulate the fracture behavior of FRP-to-concrete joints under loading/unloading cycling tests. The model is formulated within the general framework of Fracture Mechanics and is based on assuming that fracture at the FRP-to-concrete interface develops in (pure shear) mode II, as widely accepted in similar problems. Two alternative expressions of the bond-slip behavior are herein considered and their preliminary validation is finally proposed. The proposed results highlight the difference between the monotonic and the cyclic response; particularly, they show that the latter is characterized by a significantly lower force and displacement capacity.
\end{abstract}


Keywords: FRP; concrete; bond; fracture mechanics; pull-out; cyclic actions

\section{Introduction}

Fiber-reinforced polymer (FRP) materials recently gained popularity in a variety of retrofitting solutions aimed at upgrading structural members in existing civil engineering structures, such as concrete columns [1], wooden floor beams [2] and masonry panels [3]. As a matter of fact, the mechanical response of the adhesive interface often controls the structural performance of reinforced concrete (RC) members strengthened by externally-bonded (EB) FRP strips. Thus, plenty of researches aimed at investigating the bond behavior of FRP strips glued to concrete were carried out in the last decades and are currently available in the scientific literature. Particularly, the FRP-to-concrete fracture and debonding processes were thoroughly investigated via both experimental [4,5] and theoretical [6-10] studies. However, such studies, intended at investigating either the behavior of FRP-to-concrete adhesive joints or the response of EB-FRP strengthened RC beams, were generally carried out by only considering monotonic actions applied to the members under consideration.

Nevertheless, FRP strips are widely used in practical applications with the aim of enhancing the structural performance of RC beams under cyclic actions possibly induced by either traffic loads or earthquakes. Despite the significant differences between the two aforementioned load cases, the state of knowledge about the actual behavior of both the adhesive FRP-to-concrete interface and the performance of EB-FRP strengthened RC members under cyclic actions is still in need for dedicated investigations under both the experimental and theoretical standpoints. In fact, few studies are available nowadays on this topic. Particularly, some authors [11,12] reported the results of low-cycle fatigue tests carried out by assuming a single shear test set-up, whereas the results of high-cycle fatigue tests were recently documented in [13]. Regarding theoretical modeling, Ko and Sato [14] proposed an empirical bond-slip model intended at simulating the behavior observed in a series of monotonic and cyclic tests carried out on aramid (A), carbon (C) and polyacetal (P) FRP strips glued to concrete blocks and tested in double shear. The model was based on assuming a Popovics-like law and involved seven mechanical parameters, which should be calibrated experimentally, as a result of the empirical nature of the model under consideration.

This paper is intended as a further contribution to the modeling of FRP-to-concrete adhesive interface under cyclic actions: it presents a theoretical model formulated within the general framework of Fracture Mechanics (FM) to describe the post-elastic behavior of the aforementioned adhesive interface. In fact, the formulation is mainly intended at simulating the force-displacement response of FRP strips glued to a concrete substrate, as those generally adopted in single pull-out tests [15]: in principle, this tests are deemed to be representative of the debonding process which often develops at the plate end (PE) in RC beams with EB FRP strengthening in bending [16].

Although it is well known that normal stresses arising through the FRP-to-concrete interface significantly affect the behavior of FRP strips glued to concrete substrates and, then, the debonding process generally developing throughout such an interface is characterized by a mixed I/II mode of fracture [8], several theoretical studies have been recently developed by assuming that such a process 
develop in pure "mode II": this simplified assumption allows for interpreting the main aspects of the experimentally observed behavior and often results in closed-form solutions for the problem under consideration [10]. Therefore, the proposed model is also based on assuming that fracture occurs in "mode II" (i.e., pure shear) and two alternative expressions (i.e., exponential and linear softening) are considered to describe the bond stress release in the post-peak regime.

After a short literature review, Section 2 outlines the key theoretical foundations of the present model and proposes some closed-form expressions of the fracture work which can be derived once having assumed "a priori" an analytical expression (either exponential or linear) for the post-peak branch of the bond slip law. Then, Section 3 proposes some comparisons between the model simulations and a series of monotonic and cyclic test results available in the scientific literature. Concluding remarks as well as future developments of the present work are highlighted in Section 4.

Finally, in the authors' best knowledge, although several studies, both theoretical and experimental in nature, are already available to investigate the mechanical behavior of FRP-to-concrete adhesive joints under monotonic actions, no well-established formulation is available yet for simulating the cyclic response of such joints, being this topic only approached in few experimental studies, one of which is considered herein as a reference [14]. Therefore, the proposal of a mechanically based approach for simulating the cyclic response and, particularly, investigating the consequences of two different assumptions for the bond-slip law are the two main novelties of this paper, which should be intended as the first step of a new research line.

\section{Formulation of the Proposed Theoretical Model}

A simplified theoretical model is proposed to model the cyclic response of FRP strips glued to brittle substrates, made of materials such as concrete or masonry. Particularly, the present proposal is based upon the following key assumptions:

- the crack develops at the FRP-to-concrete interface in (pure shear) "mode II";

- the analytical expression of the monotonic softening branch of the bond-slip relationship is described "a priori" by assuming an analytical expression (either exponential or linear in shape);

- stiffness degradation in the unloading stages depends upon the actual value of the "fracture work" developed in each interface point;

- "small" displacements are assumed at the interface and axial strains possibly developing in the concrete substrate are neglected.

The four assumptions listed above lead to define the general governing equations for the mechanical behavior of FRP strips glued to a brittle substrate. They are derived by writing the classical "equilibrium", "compatibility" and "(generalized) stress-strain" relationships, in both monotonic and cyclic response.

\subsection{Fundamental Assumptions}

The proposed model is intended at simulating the FRP strip glued to a brittle support and schematically depicted in Figure 1. 
Figure 1. Single-lap shear test of a fiber-reinforced polymer (FRP)-to-concrete bonded joint.
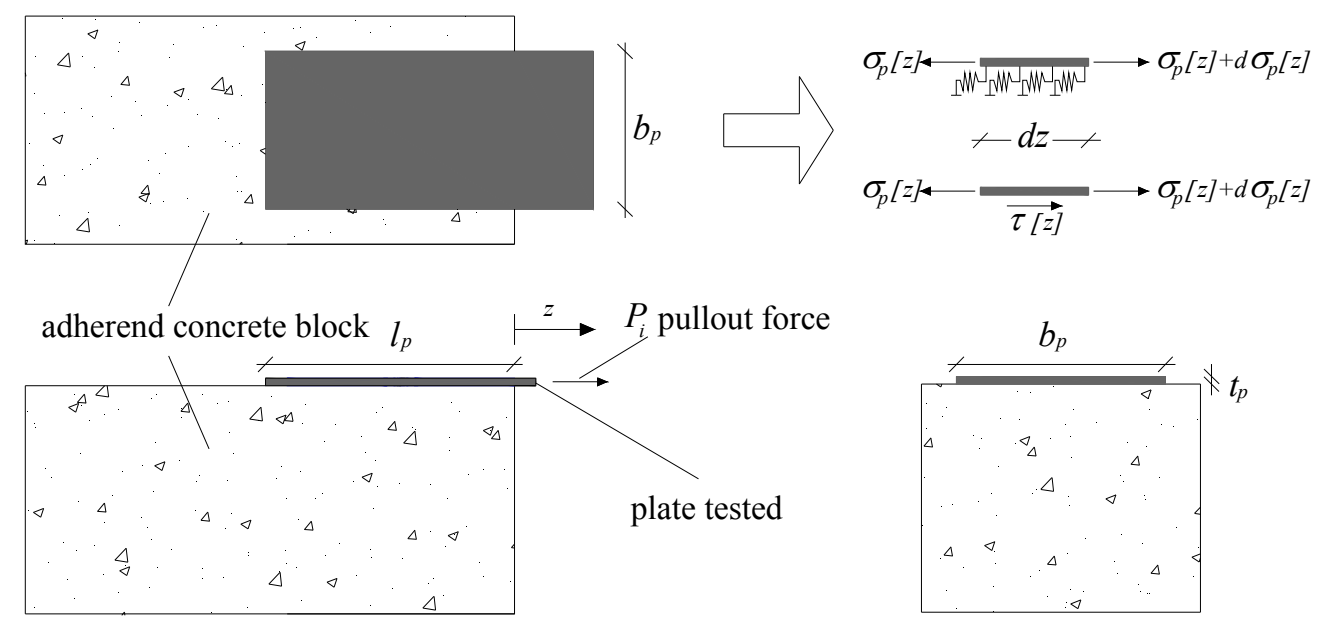

The assumptions of uniform width and thickness, $b_{p}$ and $t_{p}$, respectively, and a unique bond relationship throughout the adhesive interface, lead to the following equilibrium condition:

$$
\frac{d \sigma_{p}[z]}{d z}=-\frac{\tau[z]}{t_{p}}
$$

being $\tau[z]$ the interface bond stress and $\sigma_{p}[z]$ the axial stress in its cross section.

In this study, two alternative bond-slip laws are considered for the adhesive behavior, as discussed in Subsections 2.2.1 and 2.2.2. However, in both cases, fracture mode II is assumed.

The linear elastic behavior of the FRP strip can be easily represented by the following relationship:

$$
\sigma_{p}[z]=E_{p} \varepsilon_{p}
$$

where $E_{p}$ is the Young modulus of the composite laminate, whereas the strain field can be expressed as the first derivative of the interface slip $s[z]$ through the following compatibility equation:

$$
\varepsilon_{p}=\frac{d s[z]}{d z}
$$

Finally, the differential equation which relates the two field $s[z]$ and $\tau[z]$ can be obtained by introducing Equations (2) and (3) into the equilibrium condition [(Equation (1)]:

$$
\frac{d^{2} s[z]}{d z^{2}}+\frac{\tau[z]}{E_{p} t_{p}}=0
$$

\subsection{Fracture-Based Damage Modeling}

The unloading/reloading stiffness is modeled within the framework of fracture mechanics (FM) theory by considering, for each point of the adhesive interface, the fracture work $w_{s l}$ and the corresponding fracture energy in "mode II" $G_{f}^{I I}$. They both can be determined by assuming "a priori" a bond-slip interface law $\tau(s)$; the latter also depends on the actual cracking displacement which is assumed as a state variable. Therefore, assuming "a priori" expressions for the bond-slip laws results in several simplifications and the consistency of the numerical integration process is guaranteed 
because the value of $w_{s l}$ can be univocally defined in each point of the interface depending on the current value of the local cracking displacement.

The fracture work, developed during the sliding fracture process, controls the evolution of damage. Particularly, the variable $w_{s l}[s]$ represents the "inelastic portion" of the enclosed area of the $\tau-s$ curve in the range $[0-s]$ (Figure 2):

$$
w_{s l}[s]=\int_{0}^{s}|\tau[s]| d s-\frac{\tau^{2}[s]}{2 k_{E}}
$$

Since a unique bond-slip law, possibly defined by Equations (1) and (2), is assumed through the bond length, the value of $G_{f}^{I I}$ is uniform throughout such a length and depends on the key parameters involved in the bond-slip law under consideration:

$$
G_{F}^{I I}=\int_{0}^{\infty}|\tau[s]| d s
$$

Furthermore, a damage parameter $d$ can be defined in each point of the adhesive interface as a function of the local slip value $s=s[z]$ :

$$
d[s]=(\xi[s])^{\alpha_{d}}, \text { with } \xi[s]=\frac{w_{s l}[s]}{G_{f}^{I I}}
$$

where $\alpha_{d}$ controls the shape of the damage curve and the loading/unloading stiffness $k$ is related to the elastic one through the following relationship:

$$
k[s]=k_{E}(1-d[s])
$$

The two following subsections propose the explicit analytical expressions of Equations (5-8) corresponding to two alternative assumptions of the bond-slip law $\tau[z]$ which are considered in this study to highlight the consequences of two different expressions of the post-elastic softening branch.

\subsubsection{Exponential Softening Bond-Slip Law}

The general relationships introduced in Sections 2.1 and 2.2 can be specialized in the case of a bond-slip relationship $\tau[z]$ characterized by a negative exponential law for softening and, then, described by the following analytical expression:

$$
\tau[z]= \begin{cases}-k_{E} s[z] & \text { if } s[z] \leq s_{e} \\ -\tau_{0} e^{-\beta\left(s[z]-s_{e}\right)} & \text { if } s[z]>s_{e}\end{cases}
$$

where $k_{E}$ is the tangential bond stiffness in the pre-peak stage of the interface shear-slip relationship, $s[z]$ the interface slip at the abscissa $z, s_{e}=\tau_{0} / k_{E}$ represents the elastic slip value, $\tau_{0}$ is the shear strength, while $\beta$ is the exponential parameter of the post-peak $\tau$-s relationship (Figure 2 ). 
Figure 2. Bond-slip relationship and fracture work: exponential softening law.

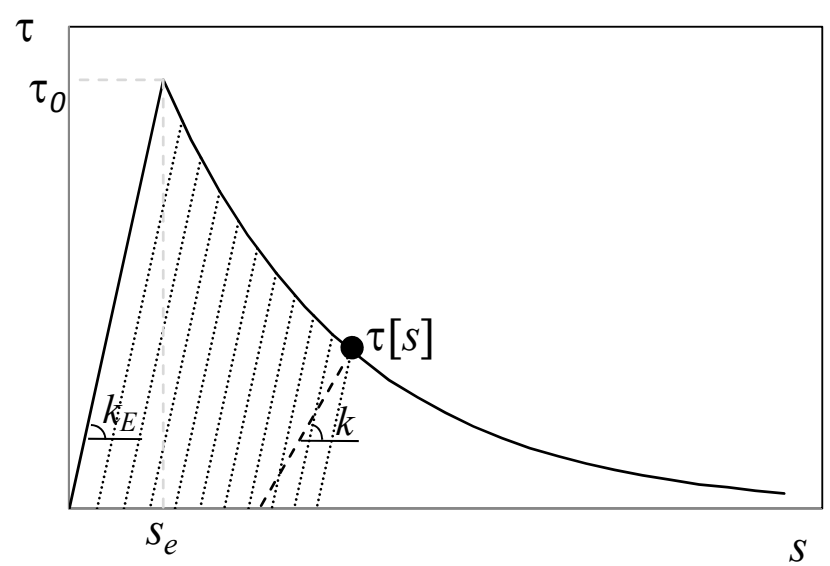

The assumption of a bond-slip law described by Equation (9) leads to the following expression for the work $w_{s l}$ dissipated in the fracture process:

$$
w_{s l}=\frac{k_{E} s_{e}^{2}}{2}\left(1-e^{-2 \beta\left(s[z]-s_{e}\right)}-\frac{2\left(e^{\beta\left(s_{e}-s[z]\right)}-1\right)}{\beta s_{e}}\right)
$$

and, clearly, $w_{s l}=0$ for $s[z]=s_{e}$.

Moreover, the corresponding value of fracture energy in mode II $G_{f}^{I I}$ can be expressed as follows, depending on the other parameters which define the assumed bond-slip law (namely $k_{E}, s_{e}$ and $\beta$ ):

$$
G_{F}^{I I}=\int_{0}^{\infty}|\tau[s]| d s=\frac{k_{E} s_{e}^{2}}{2} \cdot\left(1+\frac{2}{\beta s_{e}}\right)
$$

Figure 2 shows the unloading reloading stiffness $k$, which is clearly lower than the uncracked elastic value $k_{E}$. Its value also depends on the ratio between $w_{s l}$ and $G_{f}^{I I}$, according to the analytical expressions proposed in Equation (7) and (8).

\subsubsection{Linear Softening Bond-Slip Law}

An alternative and more common assumption for the bond-slip law $\tau[z]$ is represented by a linear softening interface model which can be alternatively defined by means of the following analytical expressions:

$$
\tau[z]= \begin{cases}-k_{E} s[z] & \text { if } s[z] \leq s_{e} \\ -\tau_{0}+k_{S}\left(s[z]-s_{e}\right) & \text { if } s_{e}<s[z] \leq s_{u} \\ 0 & \text { if } s[z]>s_{u}\end{cases}
$$

being $k_{S}$ the negative stiffness in the post-peak branch and $s_{u}=\tau_{0} / k_{E}+\tau_{0} / k_{S}$ the ultimate slip (Figure 3). Under this assumption, the work dissipated during the fracture process can be expressed as a function of the local slip $s$ through the following analytical expression:

$$
w_{s l}=\frac{\left(k_{E}+k_{S}\right)\left(s-s_{e}\right)\left(2 k_{E} s_{e}+k_{S}\left(s_{e}-s\right)\right)}{2 k_{E}} \text { with } s \leq s_{u}
$$

and, clearly, $w_{s l}=0$ for $s[z]=s_{e}$. 
Figure 3. Bond-slip relationship and fracture work: linear softening law.

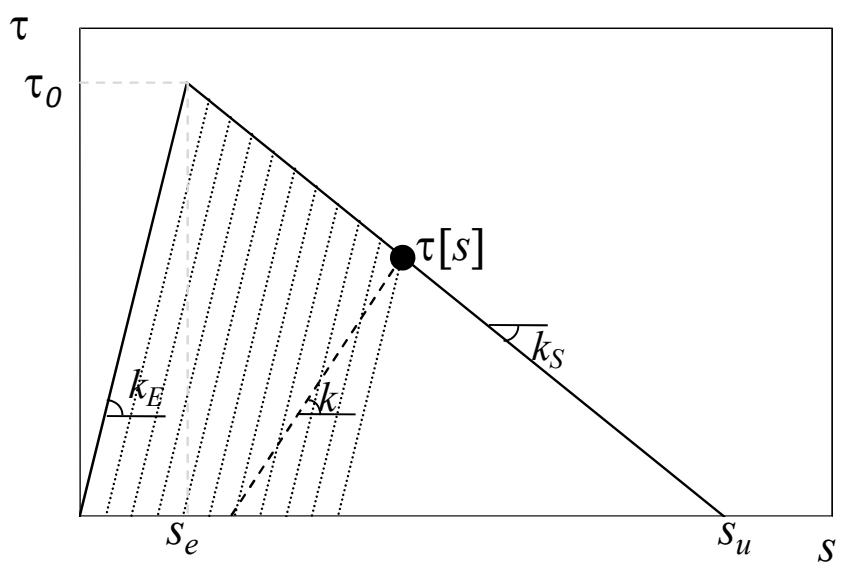

Moreover, the fracture energy can be easily expressed through the following relationship, as a function of the key parameters of the bond-slip law:

$$
G_{F}^{I I}=\int_{0}^{\infty}|\tau[s]| d s=\frac{k_{E} s_{e}^{2}}{2} \cdot\left(1+\frac{k_{E}}{k_{S}}\right)
$$

which, according to the analytical expression reported in Equation (13), is also the value achieved by $w_{s l}$ for $s=s_{u}$.

Finally, the unloading/reloading stiffness $k$, already depicted in Figure 3, can be determined once again by replacing the analytical expressions (13) and (14) of $w_{s l}$ and $G_{f}^{I I}$, respectively, within the definition of the parameters $d$ and $k$ provided by Equations (7) and (8).

\subsection{Outline of the Numerical Procedure}

A Finite Difference (FD) procedure is developed for integrating Equation (4) under monotonic and cyclic actions. Particularly, a central-difference (CD) expression is assumed to express the second derivative of Equation (4) in the internal nodes of the FD mesh represented in Figure 4:

$$
\Delta s_{i}^{j}=\frac{\Delta s_{i+1}^{j}+\Delta s_{i-1}^{j}}{2+\frac{k_{T, i}}{E_{p} t_{p}} \cdot \Delta z^{2}} \text { for } i=0, \ldots, n-1
$$

where $j$ is the current analysis step, $i$ the node number and $k_{T, i}$ the corresponding tangential stiffness of the local bond-slip law depending on the local slip value $s_{i}^{j-1}$. Since the analyses are carried out in displacement control, the following boundary conditions are applied at the unloaded and loaded end of the FRP strip, respectively:

$$
\begin{aligned}
\Delta s_{-1}{ }^{j} & =\Delta s_{1}{ }^{j} \\
\Delta s_{n}{ }^{j} & =\Delta s_{c}{ }^{j}
\end{aligned}
$$

where Equation (16) derives from the condition of zero stress (and strain) at the unloaded end, and Equation (17) corresponds the imposing the slip increment $\Delta s_{c}^{j}$ at the loaded end (i.e., node $n$ ). 
Figure 4. Finite difference discretization of the FRP-to-concrete interface.

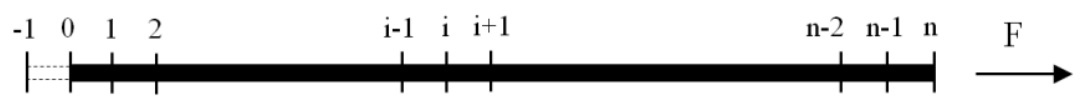

The set of $(n+2)$ simultaneous Equations (15)-(17) can be solved in terms of slip increment vector $\Delta s_{j}$ and, in principle, the final solution in the $j$-th analysis step can be obtained iteratively to take into account the possible interface nonlinearity. Particularly, the trial solution at the $k$-th iteration of the $j$-th incremental analysis step can be obtained in terms of both interface slip and bond stress vectors (which collect the $n+2$ components of both quantities):

$$
\begin{gathered}
\left.\boldsymbol{s}^{j}\right|_{k}=\boldsymbol{s}^{j-1}+\left.\Delta \boldsymbol{s}^{j}\right|_{k} \\
\left.\boldsymbol{\tau}^{j}\right|_{k}=\boldsymbol{\tau}^{j-1}+\left.\boldsymbol{\Delta} \boldsymbol{\tau}^{j}\right|_{k}=\boldsymbol{\tau}^{j-1}-\left.\boldsymbol{k}_{T} \cdot \Delta s^{j}\right|_{k}
\end{gathered}
$$

where $\boldsymbol{s}^{j-1}$ and $\boldsymbol{\tau}^{j-1}$ are slip and bond stress vectors, at the convergence of the $j$-th incremental analysis step, and $\boldsymbol{k}_{T}$ a vector collecting the tangential stiffnesses $k_{T, i}$ at the various nodes of the FD discretization (Figure 4). If the node $i$-th ended up the $(j-1)$-th analysis step in the elastic stage, the following condition should be met by the trial solution (15) for the same node to remain in elastic stage:

$$
\left|\tau_{i}^{j}\right|_{k}|\leq| \tau\left(s_{c r, i}\right) \mid
$$

where $\tau(\bullet)$ is the bond-slip law expressed by either of Equations (9) or (12) and $s_{c r, i}$ a state variable which represents the total slip developed in the node $i$ during the fracture process and, in monotonic conditions, could be simply expressed as $s_{c r, i}=s_{i}-s_{e}$. If Equation (20) is satisfied in all nodes at the first iteration $(k=1)$, then they hold their elastic status and the force $\Delta F^{j}$ increment, corresponding to the imposed slip increment $\Delta s_{c}^{j}$, can be derived by equilibrium:

$$
\left.\Delta F^{j} \stackrel{[k=1]}{=} \Delta F^{j}\right|_{k}=\left[\sum_{i=1}^{n} \frac{\left.\tau_{i}^{j}\right|_{k}+\left.\tau_{i-1}{ }^{j}\right|_{k}}{2}\right] \cdot b_{p} \cdot \Delta z
$$

If this is not the case, the slip increment $\left.\Delta s_{i}^{j}\right|_{k}$ should be subdivided in an elastic part $\left.\Delta s_{i}^{j}\right|_{k, e l}$, corresponding to the achievement of the equality in Equation (20) and the cracking part $\left.\Delta s_{i}^{j}\right|_{k, c r}=\left.\Delta s_{i}^{j}\right|_{k}-\left.\Delta s_{i}^{j}\right|_{k, e l}$. Then, an iterative search of the equilibrium for the $j$-th increment can be carried out by employing Equations (15)-(17) as a linear elastic predictor and the equality in Equation (20) to obtain the nonlinear corrector. Once convergence is achieved (i.e., in terms of unbalanced forces at the $k$-th iteration of the $j$-th increment), the vector $\boldsymbol{s}_{c r}$, whose $i$-th is the values of the state variable $s_{c r, i}$ determined in the $i$-th node, can be updated as follows:

$$
\boldsymbol{S}_{c r}{ }^{j}=\boldsymbol{S}_{c r}{ }^{j-1}+\left.\Delta \boldsymbol{s}_{c r}{ }^{j-1}\right|_{k}
$$

where $\left.\Delta \boldsymbol{s}_{c r}{ }^{j-1}\right|_{k}$ is the vector collecting all the increments of the cracking displacements $\left.\Delta s_{i}^{j}\right|_{k, c r}$ at convergence. Moreover, the corresponding force increment $\left.\Delta F^{j}\right|_{k}$ determined through Equation (21) can be added to the previous value of the external force to determine the total external load applied at the end of the $j$-th analysis step (and nodal displacement increment). 
Then, in the following incremental analysis steps, the same node $i$ will keep the cracking status if no sign change occurs between the increment slip at the previous step $(j-1)$-th and the one obtained by solving Equations (20)-(22):

$$
\left.\Delta s_{i}^{j-1} \cdot \Delta s_{i}^{j}\right|_{k}>0
$$

If this is the case for all the nodes, the corresponding force can be determined through Equation (21) and the status variable updated via Equation (22). Otherwise, an unloading stage starts in the nodes where the inequality (23) is not satisfied and an iterative predictor-corrector search leads to the new system status.

Finally, the incremental analysis proceeds up to the achievement of a given failure condition which could be practically formulated in terms of maximum slip occurring at the unloaded end.

\section{Experimental Validation}

The formulation presented in Section 2 needs to be validated in its soundness and capability to simulate the FRP-to-concrete pull-out behavior under both monotonic and cyclic conditions.

Experimental data characterizing both of the above mentioned experimental situations, on three types of FRP sheets are available in the scientific literature [14]. The results of some tests carried out on a single ply of A-FRP strips are considered to achieve a preliminary validation of the proposal.

Two groups of three nominally equal specimens were tested under monotonic and cyclic actions, respectively. They are characterized by an A-FRP strip with relative axial stiffness $E_{p} \cdot t_{p}=10.4 \mathrm{kN} / \mathrm{mm}$ and width $b_{p}=50 \mathrm{~mm}$. Then, the values of the bond-slip material parameters are identified for the two (alternative) softening laws (namely, the exponential and linear one). Particularly, $k_{E}=52.22 \mathrm{MPa} / \mathrm{mm}$, $\tau_{0}=2.256 \mathrm{MPa}$ and $G_{f}^{I I}=0.958 \mathrm{~N} / \mathrm{mm}$, are assumed in the following numerical simulations for the mechanical quantities which are relevant for both the bond-slip relationships, according to the average values identified by the cited authors for the specimens A11, A12 and A13, tested under monotonic actions. Regarding the softening branch, it can be consistently derived by the three aforementioned values and taking into account the two expressions of $G_{f}^{I I}$ described in Subsections 2.2.1 and 2.2.2 [namely, those reported as Equations (11) and (14)], which connect the $\beta$ exponent and the $k_{S}$ slope characterizing the exponential and the linear softening relationships, respectively. Moreover, the unit value is considered for the damage parameter $\alpha_{d}$.

Figure 5 compares the results (in terms of applied force-maximum slip relationship) obtained in the cyclic test labeled as "A14" in [14], with the corresponding numerical simulations obtained by assuming the exponential expression (9) for the softening branch. The agreement between experimental and numerical results is rather satisfactory, especially if it is kept in mind that no fine tuning of the relevant mechanical parameters was performed in this paper, but they were simply assumed in accordance to the values identified on monotonic tests [14]. However, the higher residual slip which affected the actual experimental observations with respect to the resulting numerical simulation points out a possible limit of the proposed fracture model which needs to be further assessed in the future stages of the validation procedure.

Finally, Figure 6 proposes a similar comparison based on the analyses carried out by assuming a linear softening branch for the bond-slip relationship described by Equation (14). 
Figure 5. Load-slip response under monotonic and cyclic actions of FRP strips glued on concrete [14]—Exponential softening.

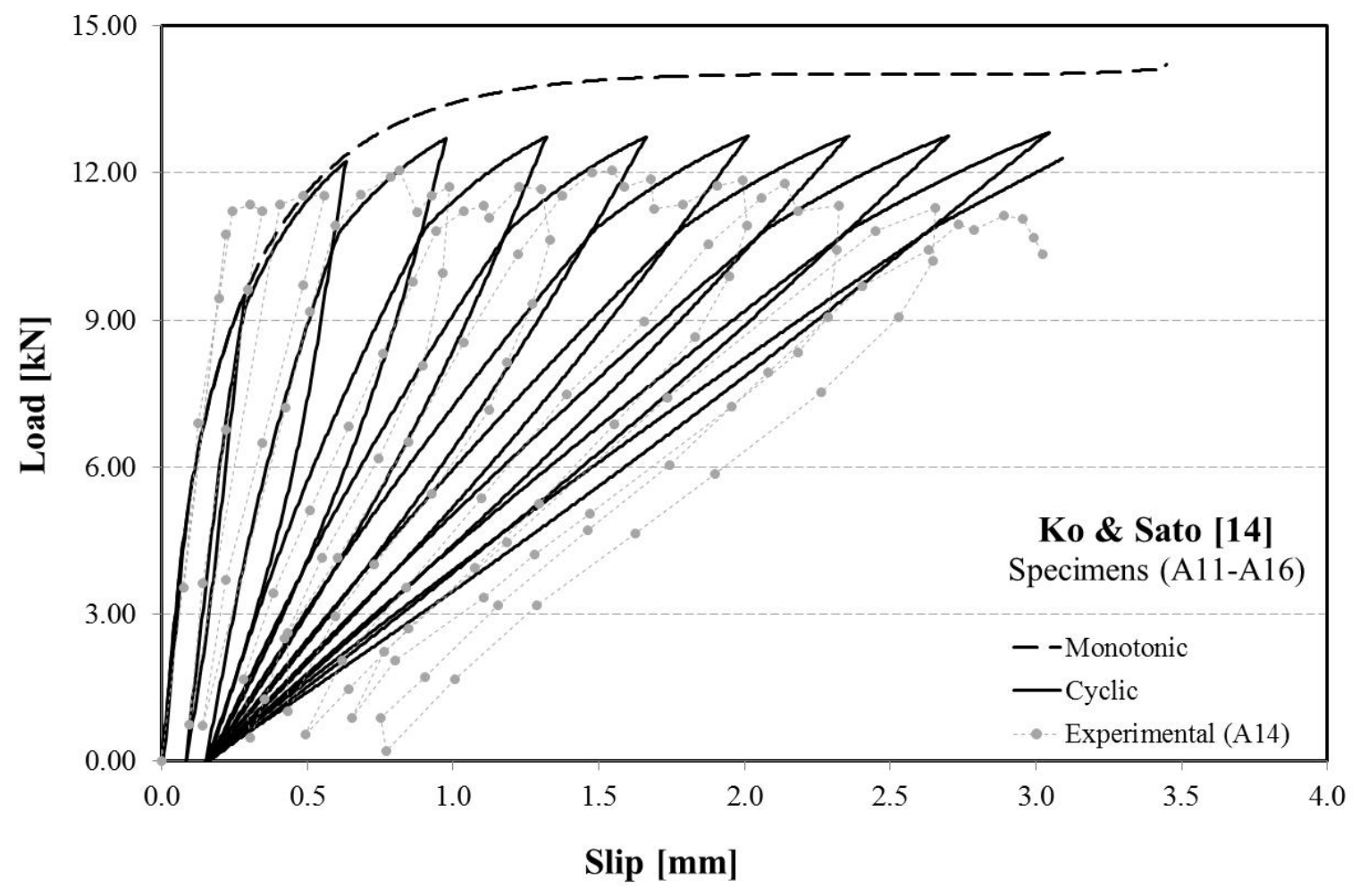

Figure 6. Load-slip response under monotonic and cyclic actions of FRP strips glued on concrete [14] — Linear softening.

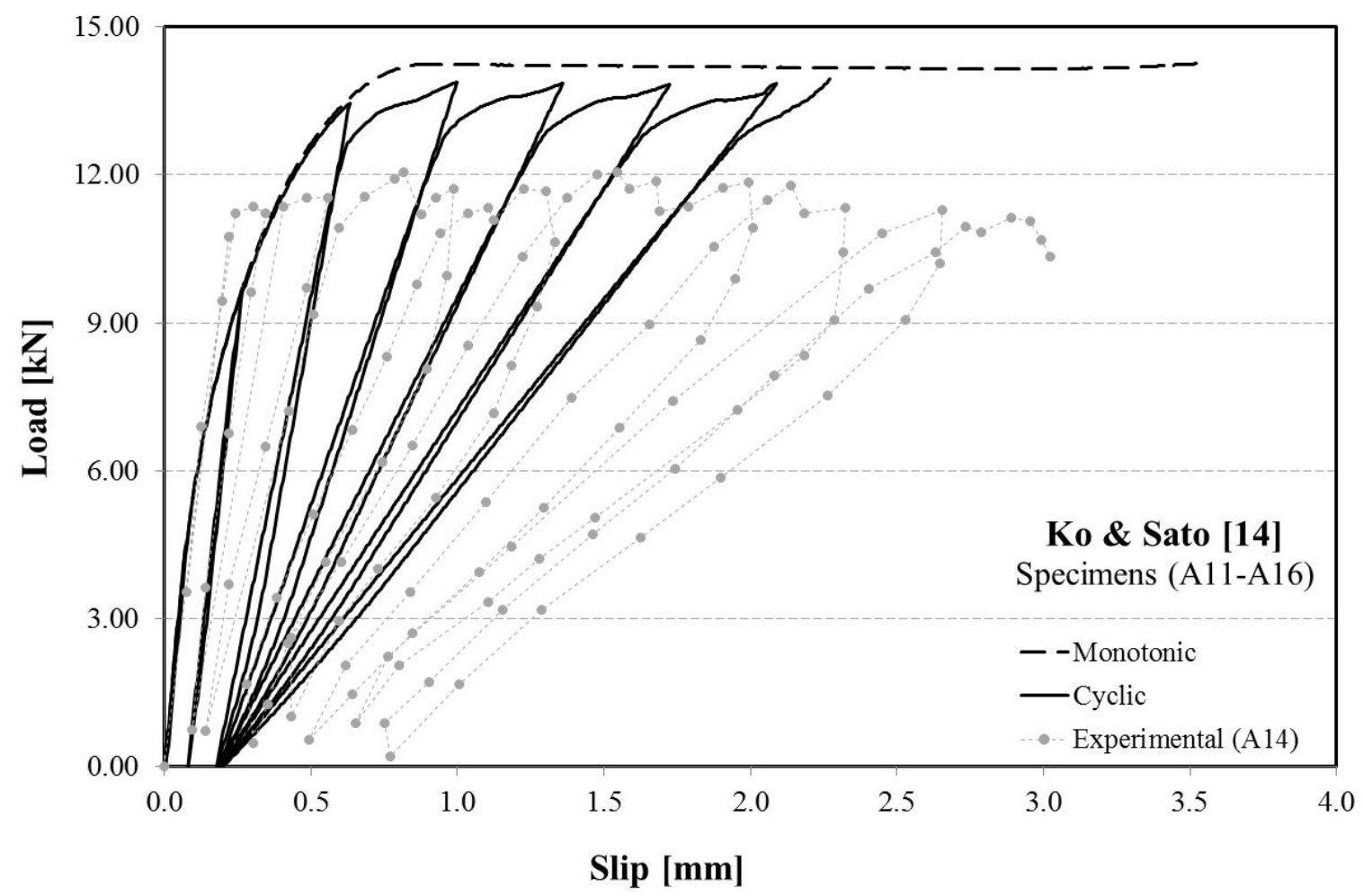


It is apparent that such an assumption, generally accepted to simulate the monotonic response of FRP strips glued to concrete, is less fit for simulating the cyclic behavior of their adhesive interface, as it generally results in an overestimation of damage which leads to debonding failure after a lower number of cycles and a smaller ultimate value of slip. Moreover, after the computational standpoint, the exponential softening law is more convenient because it simulate cracking propagation as an asymptotical process: in fact, $w_{s l}$ defined by Equation. (10) "smoothly" approaches $G_{f}^{I I}$ defined by Equation (11) and this results in an higher stability of the numerical procedure outlined in Section 2.3.

\section{Conclusions}

This paper presented a mechanically based theory and a simple numerical procedure for analyzing the debonding phenomenon which generally develops throughout the interface of FRP plate glued on concrete substrates. The following comments can be finally highlighted:

- the proposed model has been formulated within the framework of Fracture Mechanics and assumed two alternative expressions for the softening branch of the bond-slip relationship;

- the closed-form expressions obtained for determining the key fracture-related quantities are among the novel and most attractive features of the present formulation;

- the comparison between some experimental results available in the literature and the numerical simulations performed by means of the present model highlighted its high predictive potential;

- the proposed experimental comparisons pointed out the higher accuracy obtained by assuming an exponential softening branch, with respect to the linear one, generally accepted for simulating the response under monotonic actions;

- the extension of such comparisons to further experimental observation is among the future steps of this research, that finally aims at characterizing the force and displacement capacity of FRP-to-concrete joints subjected to cyclic actions, which are quite common in seismic retrofitting of existing buildings.

\section{Acknowledgments}

This work was realized during the visit of the second co-author at the University of Salerno (Italy), as part of the "EnCoRe" Project (FP7-PEOPLE-2011-IRSES no. 295283; www.encore-fp7.unisa.it) financed by the European Union, whose contribution is gratefully acknowledged.

\section{Conflicts of Interest}

The authors declare no conflict of interest.

\section{References}

1. Pan, J.L.; Xu, T.; Hu, Z.J. Experimental investigation of load carrying capacity of the slender reinforced concrete columns wrapped with FRP. Constr. Build. Mater. 2007, 21, 1991-1996.

2. Corradi, M.; Speranzini, E.; Borri, A.; Vignoli, A. In-plane shear reinforcement of wood beam floors with FRP. Compos. Part B Eng. 2006, 37, 310-319. 
3. Marcari, G.; Manfredi, G.; Prota, A.; Pecce, M. In-plane shear performance of masonry panels strengthened with FRP. Compos. Part B Eng. 2007, 38, 887-901.

4. Chajes, M.; Finch, W.; Januska, T.; Thomson, T. Bond and force transfer of composite material plates bonded to concrete, ACI Struct. J. 1996, 93, 208-217.

5. Czaderski, C.; Martinelli, E.; Michels, J.; Motavalli, M. Effect of curing conditions on strength development in an epoxy resin for structural strengthening. Compos. Part B Eng. 2012, 43, 398-410.

6. Ferracuti, B.; Savoia, M.; Mazzotti, C. A numerical model for FRP-concrete delamination. Compos. Part B Eng. 2006, 37, 356-364.

7. Cornetti, P.; Carpinteri, A. Modelling the FRP-concrete delamination by means of an exponential softening law. Eng. Struct. 2001, 33, 1988-2001.

8. Martinelli, E.; Czaderski, C.; Motavalli, M. Modeling in-plane and out-of-plane displacement fields in pull-off tests on FRP strips. Eng. Struct. 2011, 33, 3715-3725.

9. Caggiano, A.; Martinelli, E.; Faella, C. A fully-analytical approach for modelling the response of FRP plates bonded to a brittle substrate. Int. J. Solids Struct. 2012, 49, 2291-2300.

10. Caggiano, A.; Martinelli, E. A fracture-based interface model for simulating the bond behaviour of FRP strips glued to a brittle substrate. Compos. Struct. 2013, 99, 397-403.

11. Mazzotti, C.; Savoia, M. FRP-concrete bond behaviour under cyclic debonding force. Adv. Struct. Eng. 2009, 12, 771-780.

12. Nigro, E; di Ludovico, M.; Bilotta, A. Experimental investigation of FRP-concrete debonding under cyclic actions. J. Mater. Civil Eng. (ASCE) 2011, 23, 360-371.

13. Carloni, C.; Subramaniam, K.V.; Savoia, M.; Mazzotti, C. Experimental determination of FRP-concrete cohesive interface properties under fatigue loading. Compos. Struct. 2012, 94, 1288-1296.

14. Ko, H.; Sato, Y. Bond stress-slip relationship between FRP sheet and concrete under cyclic load. J. Compos. Construct. (ASCE) 2007, 11, 419-246.

15. Fédération Internationale du Béton (FIB). Bulletin 14: Externally Bonded FRP Reinforcement for RC Structures; Technical report, ISBN 978-2-88394-054-3; FIB: Lausanne, Switzerland, 2001.

16. Martinelli, E.; Bilotta, A.; Faella, C.; Nigro, E. On the Behavior of FRP-to-concrete Adhesive Interface: Theoretical Models and Experimental Results. In Advances in Composite Materials-Ecodesign and Analysis; Attaf, B., Ed.; InTech Open Access Publisher: Rijeka, Croatia, 2011; pp. 517-546.

(C) 2014 by the authors; licensee MDPI, Basel, Switzerland. This article is an open access article distributed under the terms and conditions of the Creative Commons Attribution license (http://creativecommons.org/licenses/by/3.0/). 